\title{
Research and Application of Fire Forecasting Model for Electric Transmission Lines Incorporating Meteorological Data and Human Activities
}

\author{
Jiazheng Lu, ${ }^{1,2}$ Jun Guo, ${ }^{1,2}$ Li Yang, ${ }^{1,2}$ Tao Feng, ${ }^{1,2}$ and Jie Zhang ${ }^{1,2}$ \\ ${ }^{1}$ State Key Laboratory of Disaster Prevention and Reduction for Power Grid Transmission and Distribution Equipment, \\ Changsha 410129, China \\ ${ }^{2}$ State Grid Hunan Electric Power Company Disaster Prevention and Reduction Center, Changsha 410129, China
}

Correspondence should be addressed to Jiazheng Lu; lujz1969@126.com

Received 12 March 2016; Accepted 29 September 2016

Academic Editor: Marco Mussetta

Copyright (C) 2016 Jiazheng Lu et al. This is an open access article distributed under the Creative Commons Attribution License, which permits unrestricted use, distribution, and reproduction in any medium, provided the original work is properly cited.

Recently, there is a rise in frequency of fires which pose a serious threat to the safety operation of electric transmission lines. Several ultrahigh voltage (UHV) electric transmission lines, including Fufeng line, Jinsu line, Longzheng line, and Changnan line, showed many times tripping or bipolar latching caused by fire disasters. Fire disasters have tended to be the biggest threat to the safety operation of electric transmission lines and even can cause power grid collapse in some severe situations. Researchers have made much research on fires forecasting. However, these studies are mainly concentrated on predicting fires based on measured or forecasting meteorological data and do not take into account the effect of human activities. In fact, fire disasters have a very close relationship with human activities. In our research, a fire prediction model is proposed incorporating meteorological data as well as human activities. And this model is applied in Hunan province and Anhui province, which seriously suffer from fire disasters. The results show that the model has good prediction precision and can be a powerful tool for practical application.

\section{Introduction}

In recent years, with the implementation of "returning farmland to forest" policy of the Chinese Government and the effect of the conventional custom of using fire, the frequency of fires, which pose a serious threat to the safety operation of electric transmission lines, is on the rise. In the year 2013, several ultrahigh voltage (UHV) transmission lines, including Fufeng line, Jinsu line, Longzheng line, and Changnan line, showed many times tripping or bipolar latching caused by fire disasters. Fire disasters have tended to be the biggest threat to the safety operation of electric transmission lines and even can cause power grid collapse in some severe situations. Therefore, it is urgent to carry out studies on fire disasters to provide technical support to safety operation of electric transmission lines.

Researchers have made much research on fires forecasting. The earliest research can be dated back to the early 20th century. In the year 1975, America developed the first automatic fire forecasting system: Administrative and Forest Fire Information Retrieval and Management System (AFFIRMS). Burgan [1] employed satellite and surface observations to estimate the characteristics of fire disasters $[1,2]$. Vega-Garcia et al. [3] used artificial neural networks to predict humancaused wildfire. Also, Wen et al. [4] and $\mathrm{Hu}$ et al. [5] predicted fires by artificial neural networks. Fu et al. [6] constructed a fire forecasting model based on the Grey theory. Stojanova et al. [7] applied several data mining techniques (logistic regression, random forest (RF), and decision trees (DT)) to predict fires. Cortez and Morais [8] adopted support vector machine (SVM) for fire forecasting and only four typical meteorological predictors are taken into account in their model.

However, these studies are more concentrated on constructing a fire forecasting model based on measured or forecasting meteorological data or remote sensing data. In 


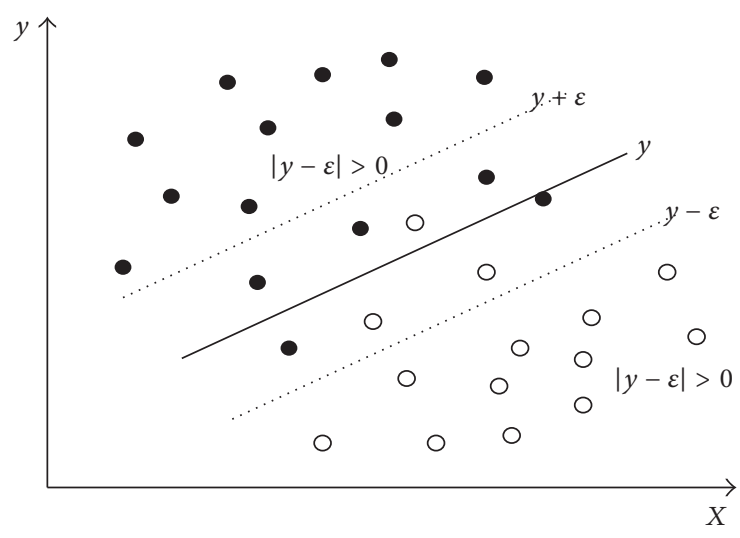

FIGURE 1: SVM for classification with $\varepsilon$-insensitive tube.

fact, fire disasters have a very close relationship with human activities, especially the conventional custom of using fire during traditional Chinese festivals. Therefore, the human effect should be taken into account in the fire forecasting model. In this paper, we proposed a fire forecasting model based on meteorological data as well as human activities.

The remainder of this paper is organized as follows: Section 2 presents several basic theories referred to in our paper; Section 3 describes the methods employed in model construction and model estimation; Section 4 gives detailed information about the case study; Section 5 demonstrates the results and discussion about the case study; and conclusions are made in Section 6.

\section{Basic Theories}

2.1. Support Vector Machine (SVM) for Classification. $\left\{X_{i}, y_{i}\right\}$ is the training sample, where $i=1,2, \ldots, l, X_{i}$ denotes the input, and $y_{i}$ is the corresponding output. The aim of SVM for classification is to find the hyperplane to classify the sample data into two parts (shown in Figure 1).
This hyperplane can be expressed as a function of this form:

$$
y_{i}=W \cdot X_{i}+b
$$

where $W$ is a hyperplane and $b$ is the offset. follows:

The SVM for classification will use a penalty function as

$$
\begin{aligned}
& \left|y_{i}-\left(W \cdot X_{i}+b\right)\right| \leq \varepsilon, \quad \text { not allocating a penalty, } \\
& \left|y_{i}-\left(W \cdot X_{i}+b\right)\right|>\varepsilon, \quad \text { allocating a penalty. }
\end{aligned}
$$

The bound by $y_{i} \pm \varepsilon$ is called an $\varepsilon$-insensitive tube. And the optimization problem can be expressed as

$$
\min \left[\frac{1}{2}\|W\|^{2}+C \sum_{i=1}^{l} L^{\varepsilon}\left(X_{i}, y_{i}, f\right)\right]
$$

where $L^{\varepsilon}\left(X_{i}, y_{i}, f\right)$ is defined as

$$
L^{\varepsilon}\left(X_{i}, y_{i}, f\right)=\max \left(0,\left|f\left(X_{i}\right)-y_{i}\right|-\varepsilon\right) .
$$

And the slack variables $\xi^{+}$and $\xi^{-}$are employed to handle the errors in classification; then, the form of SVM for classification will be

$$
\min \left[\frac{1}{2}\|W\|^{2}+C \sum_{i=1}^{l}\left(\xi_{i}^{+}+\xi_{i}^{-}\right)\right]
$$

Subject to: $\quad\left(W \cdot X_{i}+b\right)-y_{i} \leq \varepsilon+\xi^{+}$

$$
\begin{aligned}
& y_{i}-\left(W \cdot X_{i}+b\right) \leq \varepsilon+\xi^{-} \\
& \xi^{+}>0 \\
& \xi^{-}>0 \\
& i=1,2, \ldots, l .
\end{aligned}
$$

The corresponding dual problem can be derived as

$$
\max \left[\sum_{i=1}^{l}\left(\alpha_{i}^{+}-\alpha_{i}^{-}\right) y_{i}-\varepsilon \sum_{i=1}^{l}\left(\alpha_{i}^{+}+\alpha_{i}^{-}\right)-\frac{1}{2} \sum_{i, j}\left(\alpha_{i}^{+}-\alpha_{i}^{-}\right)\left(\alpha_{j}^{+}-\alpha_{j}^{-}\right) X_{i} \cdot X_{j}\right]
$$

Subject to: $0 \leq \alpha_{i}^{+} \leq C$,

$0 \leq \alpha_{i}^{-} \leq C$

$$
\sum_{i=1}^{l}\left(\alpha_{i}^{+}-\alpha_{i}^{-}\right)=0
$$

$i=1,2, \ldots, l$. 
However, this model is only suitable for a linear problem. And then the kernel function is introduced to circumvent this problem. The kernel function can transform the low dimension space into high dimension space. Then, this model for classification will be feasible for the high dimension space. Thereafter, the goal function of the above form will be explained as

$$
\max \left[\sum_{i=1}^{l}\left(\alpha_{i}^{+}-\alpha_{i}^{-}\right) y_{i}-\varepsilon \sum_{i=1}^{l}\left(\alpha_{i}^{+}+\alpha_{i}^{-}\right)-\frac{1}{2} \sum_{i, j}\left(\alpha_{i}^{+}-\alpha_{i}^{-}\right)\left(\alpha_{j}^{+}-\alpha_{j}^{-}\right) K\left(X_{i}, X_{j}\right)\right],
$$

where $K\left(X_{i}, X_{j}\right)$ is the Kernel function. Usually, the radial basis kernel $K\left(X_{i}, X_{j}\right)=\exp \left(-\left\|X_{i}-X_{j}\right\|^{2} / 2 \sigma^{2}\right)$ is adopted, and we also use this kernel function in our paper.

2.2. Differential Evolution (DE) Algorithm. DE algorithm was firstly proposed by Storn and Price in the year 1995 $[9,10]$, and it is used for global optimization over continuous space. This algorithm is built based on stochastic searching. The philosophy behind this algorithm is very simple and this algorithm can be easily implemented by computer programming. Therefore, it has been widely used for applications since it was proposed [1115]. The framework of the DE algorithm is described as follows.

The DE algorithm is evolving based on a population which consists of $N_{p}$ individuals as $x_{i}^{g}, i=1,2, \ldots, N_{p}$, where $g$ denotes the current times of generation. If the problem is related to $D$ parameters, then each individual is a $D$-dimension vector, and each dimension is related to the corresponding parameter.

Based on the population, $N_{p}$ mutant individuals are generated according to

$$
v_{i}^{g+1}=x_{r 1}^{g}+F\left(x_{r 2}^{g}-x_{r 3}^{g}\right), \quad i=1,2, \ldots, N_{p},
$$

where $x_{r 1}^{g}, x_{r 2}^{g}$, and $x_{r 3}^{g}$ are three different individuals randomly chosen from the population $x_{i}^{g}$ and $F$ is the mutation control parameter that controls the generation of the differential vector.

After mutation, the crossover operator is applied to the mutant individuals and population $x_{i}^{g}$ as follows:

$$
\begin{aligned}
& u_{i, j}^{g+1} \\
& = \begin{cases}v_{i, j}^{g+1}, & \text { if }((\operatorname{random}()<\mathrm{CR}) \text { or }(j=\operatorname{random}(1, D))) \\
x_{i, j}^{g+1}, & \text { otherwise, }\end{cases}
\end{aligned}
$$

where $u_{i}^{g+1}=\left(u_{i, 1}^{g+1}, u_{i, 2}^{g+1}, \ldots, u_{i, D}^{g+1}\right), i=1,2, \ldots, N_{p}$ are $N_{p}$ new generated individuals, CR is the crossover parameter, random( ) denotes a float type number chosen between 0 and 1 , and random $(1, D)$ denotes an integer type number chosen between 1 and $D$.
At last, the selection operator is activated. DE algorithm adopts the greedy selection strategy as follows:

$$
x_{i}^{g+1}= \begin{cases}u_{i}^{g+1}, & \text { if }\left(u_{i}^{g+1} \text { is better than } x_{i}^{g}\right) \\ x_{i}^{g}, & \text { otherwise, }\end{cases}
$$

where $x_{i}^{g+1}$ is the generated next population.

2.3. Improved $D E$ Algorithm. It is known that the $\mathrm{DE}$ algorithm can easily fall into local optimum and cannot converge to the global optimum. Therefore, the improved $\mathrm{DE}$ algorithm, the adaptive differential evolution algorithm combined with chaotic search (CADE), proposed by Lu et al. [16], is adopted for model optimization. And the CADE algorithm is briefly described as follows.

(1) CADE algorithm introduces the adaptive crossover parameter as

$$
\mathrm{CR}=\mathrm{CR}_{0} \cdot 2^{\exp \left(1-g_{\max } /\left(g_{\text {now }}+1\right)\right)},
$$

where $\mathrm{CR}_{0}$ is the constant crossover parameter; $g_{\max }$ is the maximum number of generations; $g_{\text {now }}$ denotes the current number of generations; CR is the adaptive crossover parameter.

Wu et al. [17] stated that the DE algorithm has strong local search ability with big CR value while the $\mathrm{DE}$ algorithm is more concentrated on global searching with small CR value. Thus, the adaptive crossover parameter CR can adaptively control the evolution of the algorithm and set small CR at the beginning and large CR value at the end.

(2) CADE algorithm introduces the chaotic searching operator.

The chaotic searching operator has good performance of global traversals. Thus, it can be employed to improve the algorithm search precision. The chaotic searching operator is described as follows.

Assume the original individual is $X=\left[x_{1}, x_{2}, \ldots, x_{D}\right]$. We can get $K$ individuals as follows:

$$
\begin{aligned}
X^{\prime} & =\left[x_{1}^{\prime}, x_{2}^{\prime}, \ldots, x_{D}^{\prime}\right], \\
x_{j}^{\prime} & =\left(1-u_{g}\right) x_{j}+u_{g} P_{j}, \\
P_{j} & =x_{j}^{\min }+r_{j}^{k}\left(x_{j}^{\max }-x_{j}^{\min }\right),
\end{aligned}
$$




$$
\begin{aligned}
& r_{j}^{k+1}=\lambda \cdot r_{j}^{k}\left(1-r_{j}^{k}\right), \\
& u_{g}=\exp \left(-m \cdot \frac{g_{\text {now }}}{g_{\max }}\right) \\
& \quad j=1,2, \ldots, D, k=1,2, \ldots, K, r_{j} \in[0,1],
\end{aligned}
$$

where $\lambda$ is the chaotic parameter and is set to be 4 ; $X^{\prime}$ is the generated individual; $m$ is the scale factor.

This operator has two parameters: the amount of chaotic searching $K$ and the scale factor $m$. For more details, readers can refer to Lu et al. [16].

\section{Methods}

3.1. Human Index. It is known that fires are always associated with human activities. Therefore, to precisely predict fires, the human factor should be paid more attention. Based on the analysis of fires data and practical experience of fire prevention of electric power transmission lines, we found that the fires are usually happening near the Chinese festivals and farming activities, including Ching Ming Festival, Chinese New Year, Ghost Festival, spring planting, and autumn harvest, due to the ancestor worship customs in these Chinese festivals and grass burning of farming activities. At the same time, the outbreak of fires has a prerequisite; that is, no rain exists during these Chinese festivals and farming activities. In this paper, a human index is proposed for fires forecasting as follows:

$$
\mathrm{HI}=\alpha \cdot e^{-p}
$$

where $\mathrm{HI}$ is the proposed human index; $p$ is the precipitation value; $\alpha$ is the coefficient related to human activities and is assigned as follows:

$\alpha$

$$
= \begin{cases}1, & \text { Ching Ming Festival or Chinese New Year } \\ 0.7, & \text { Ghost Festival } \\ 0.4, & \text { autumn harvest } \\ 0.3, & \text { spring planting } \\ 0.1, & \text { otherdays. }\end{cases}
$$

3.2. Evaluation Index. The evaluation index is used to estimate the precision of fire forecasting. As the fires disposition measures are chosen based on the number of fires, the fire forecasting model is constructed to predict the number of daily fires. In this paper, the severity of fires is classed into 4 levels (level 1: 0-50 fires; level 2: 51-150 fires; level 3: 151250 fires; level 4: more than 251 fires). The accuracy rate is always adopted to estimate the precision of the fire forecasting model. However, the 4 levels do not have equal importance. It is obvious that more attention should be paid to level 4 . Therefore, a weighted accuracy rate index is raised as follows:

$$
R_{w}=\lambda_{1} \cdot R_{1}+\lambda_{2} \cdot R_{2}+\lambda_{3} \cdot R_{3}+\lambda_{4} \cdot R_{4},
$$

where $R_{w}$ is the weighted accuracy rate index; $R_{i}$ is the accuracy rate of level $i ; \lambda_{i}$ is the weight coefficient for level $i$; it has to be noted that the bigger weight coefficient should be assigned to higher level.

3.3. Optimization of SVM Model. The calibration of fire forecasting model has a huge influence on the precision of fire prediction. In this paper, the global optimization algorithm CADE is employed for model calibration. Otherwise, before model calibration, we have to determine the optimal model structure. In this paper, this means choosing optimal predictors. There are many ways of determining the optimal model structure. The trial-and-error method is the most popular method. However, this method is time-consuming and needs a large amount of computation. The method proposed by Guo et al. [18] is adopted to improve the efficiency. This method can optimize the parameters of SVM model and choose optimal predictors simultaneously. For convenience, we name this method Guo's method, and Guo's method is briefly described as follows:

(1) Select potential predictors. Several potential predictors are chosen based on data analysis or experiences.

(2) Encode potential predictors. Each potential predictor is assigned a float number in the range $[0,1]$ and is named predictor parameter. When the predictor parameter is larger than 0.5 , this means that the predictor associated with the predictor parameter has positive contribution to model prediction; otherwise, this will mean that this predictor has a negative effect on model prediction.

(3) Combine the parameters of SVM model and encode predictors. It is known that the SVM model usually contains three parameters: penalty coefficient $C$, slack coefficient $\varepsilon$, and kernel function parameter $\sigma$. And each potential predictor can be treated as a parameter. If there are $n$ potential predictors, then the total number of parameters of SVM model will be $n+3$.

(4) Optimize the parameters of SVM model. With this definition, we can optimize the parameters of SVM model and choose optimal predictors simultaneously.

\section{Case Study}

4.1. Descriptions of the Study Area. To test the accuracy of the fire forecasting model, two provinces of China, that is, Hunan province and Anhui province, are chosen as the study areas in our research. These two provinces suffered the heaviest fire disasters in China. The fire data of these two provinces is available from 1 January 2001 to 31 December 2012 and is shown in Figures 2 and 3. The features of fire disasters in these provinces are summarized in Table 1 . It can be noted that (1) Anhui province has the largest number of fires; (2) fire disasters tend to be more and more serious; (3) fire disasters are concentrated at some specific point of time.

4.2. Parameter Sets of Optimization Algorithm. The parameter sets of CADE algorithm are as follows: the size of the 


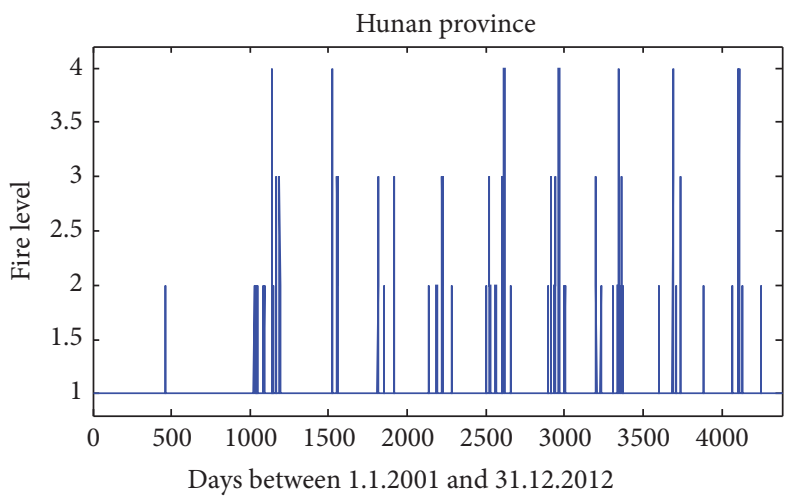

FIGURE 2: Fire data of Hunan province.

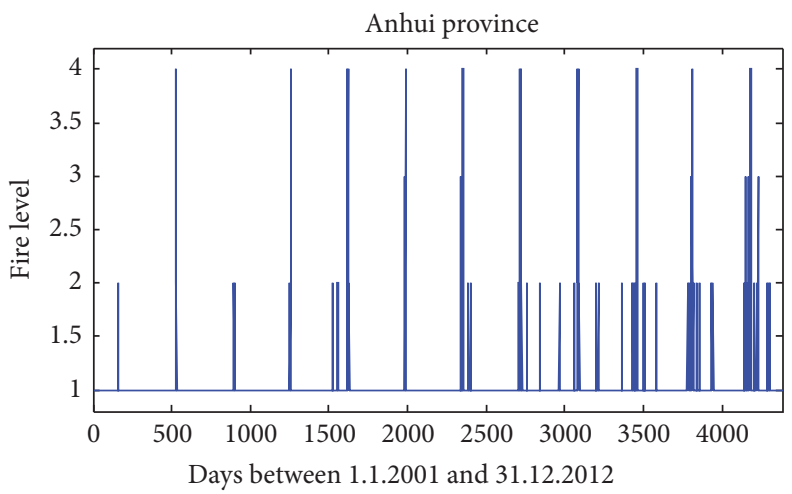

FIgURE 3: Fire data of Anhui province.

TABLE 1: The features of fire disasters in two provinces.

\begin{tabular}{lcc}
\hline Province & Hunan & Anhui \\
\hline Maximum number of fires & 1536 & 2587 \\
Minimum number of fires & 0 & 0 \\
Average number of fires & 7.73 & 9.21 \\
\hline
\end{tabular}

population is set to 100 ; the constant crossover parameter $\mathrm{CR}_{0}$ is set to 0.3 ; the scale factor $m$ of the local chaos searching operator is set to 8; the maximum number of generations is set to 1000; and the maximum iteration number of the local chaos searching $K$ is set to 150 . The parameters of the weighted accuracy rate index are set as follows: $\lambda_{1}=1, \lambda_{2}=2, \lambda_{3}=4$, and $\lambda_{4}=8$. The optimization range of the parameter penalty coefficient $C$ is set to $[0.001,200]$, the optimization range of the parameter kernel function parameter $\sigma$ is set to $[0,5]$, and the optimization range of the parameter slack coefficient $\varepsilon$ is set to $[0,0.5]$.

4.3. Comparative Framework. First, the fire forecasting model is constructed based on the method described in Section 3.3. Moreover, to reveal the effect of the predictors on fire forecasting, the forecasting model with different combinations of predictors is comparatively analyzed. It can be noted from Figures 2 and 3 that the fire disasters of the later 6 years are obviously more serious than those of the previous
6 years. Therefore, to ensure the training data set contains all of the extreme events of fire disasters, the later 6 years' data is selected as the training data, while the other is treated as the validation data.

\section{Results and Discussion}

It is known that the model optimization algorithm is evolving based on random searching mechanism. To reduce the effect of the random factors in this algorithm, the algorithm runs 10 times independently in each trial.

First of all, we select several potential predictors associated with fire disasters. These potential predictors are chosen based on our practical experiences and mainly include daily minimum air temperature, daily maximum air temperature, daily mean air temperature, daily precipitation, daily minimum relative air humidity, and daily mean relative air humidity.

Then, several typical forecasting models are constructed as follows: (1) the predictors are daily maximum air temperature and daily precipitation; (2) the predictors are daily maximum air temperature, daily precipitation, and daily minimum relative air humidity; (3) the predictors are daily mean air temperature, daily precipitation, and daily mean relative air humidity; (4) the predictors contain all of the listed potential predictors; (5) the predictors of the model are optimized by the CADE algorithm. 
TABLE 2: The results of typical models without the human index in Hunan province.

\begin{tabular}{|c|c|c|c|c|c|c|}
\hline & Index & Model 1 & Model 2 & Model 3 & Model 4 & Model 5 \\
\hline \multirow{6}{*}{ Training } & Traditional accuracy rate & 0.9658 & 0.9676 & 0.9662 & 0.9804 & 0.9804 \\
\hline & Weighted accuracy rate & 0.9658 & 0.9767 & 0.9694 & 1.0356 & 1.0356 \\
\hline & Traditional accuracy rate of fire level 1 & 1.0 & 1.0 & 1.0 & 0.9995 & 0.9995 \\
\hline & Traditional accuracy rate of fire level 2 & 0 & 0 & 0 & 0.2826 & 0.2826 \\
\hline & Traditional accuracy rate of fire level 3 & 0 & 0.1333 & 0 & 0.5333 & 0.5333 \\
\hline & Traditional accuracy rate of fire level 4 & 0 & 0.1428 & 0.0714 & 0.8571 & 0.8571 \\
\hline \multirow{6}{*}{ Test } & Traditional accuracy rate & 0.9795 & 0.9799 & 0.9795 & 0.9795 & 0.9795 \\
\hline & Weighted accuracy rate & 0.9795 & 0.9813 & 0.9795 & 0.9840 & 0.9840 \\
\hline & Traditional accuracy rate of fire level 1 & 1.0 & 1.0 & 1.0 & 0.9981 & 0.9981 \\
\hline & Traditional accuracy rate of fire level 2 & 0 & 0 & 0 & 0.0909 & 0.0909 \\
\hline & Traditional accuracy rate of fire level 3 & 0 & 0.1 & 0 & 0 & 0 \\
\hline & Traditional accuracy rate of fire level 4 & 0 & 0 & 0 & 0.5 & 0.5 \\
\hline
\end{tabular}

TABLE 3: The results of typical models without the human index in Anhui province.

\begin{tabular}{|c|c|c|c|c|c|c|}
\hline & Index & Model 1 & Model 2 & Model 3 & Model 4 & Model 5 \\
\hline \multirow{6}{*}{ Training } & Traditional accuracy rate & 0.9539 & 0.9557 & 0.9544 & 0.9594 & 0.9594 \\
\hline & Weighted accuracy rate & 0.9539 & 0.9658 & 0.9576 & 0.9891 & 0.9891 \\
\hline & Traditional accuracy rate of fire level 1 & 1 & 1 & 1 & 0.9995 & 0.9995 \\
\hline & Traditional accuracy rate of fire level 2 & 0 & 0.0167 & 0 & 0.05 & 0.05 \\
\hline & Traditional accuracy rate of fire level 3 & 0 & 0 & 0 & 0.1429 & 0.1429 \\
\hline & Traditional accuracy rate of fire level 4 & 0 & 0.1111 & 0.0370 & 0.2963 & 0.2963 \\
\hline \multirow{6}{*}{ Test } & Traditional accuracy rate & 0.9836 & 0.9831 & 0.9836 & 0.9781 & 0.9781 \\
\hline & Weighted accuracy rate & 0.9836 & 0.9895 & 0.9836 & 0.9813 & 0.9813 \\
\hline & Traditional accuracy rate of fire level 1 & 1 & 0.9986 & 1 & 0.9940 & 0.9940 \\
\hline & Traditional accuracy rate of fire level 2 & 0 & 0 & 0 & 0 & 0 \\
\hline & Traditional accuracy rate of fire level 3 & 0 & 0 & 0 & 0 & 0 \\
\hline & Traditional accuracy rate of fire level 4 & 0 & 0.25 & 0 & 0.125 & 0.125 \\
\hline
\end{tabular}

For convenience, we call these models model 1, model 2, model 3, model 4, and model 5, respectively. The results of the above 5 models are summarized in Tables 2 and 3. Similar to [19], Figure 4 shows the results of each trial related to models without the human index. From the results, the following can be noted:

(1) Model 2 has better performance than model 1. This indicates that air humidity has positive contribution to fire forecasting.

(2) Model 3 is worse than Model 2. Besides, model 3 has lower forecasting precision than model 2 on fire level 3 and level 4 . This indicates that the mean air temperature and mean relative air humidity may not be representative compared to maximum air temperature and minimum relative air humidity.

(3) Model 4 and model 5 get the same results, and they have the best performance among these 5 models. For model 5, the calibration results of the predictor parameters are all larger than 0.5 . This means that these 6 potential predictors are all necessary for fire forecasting. Also, we can confirm this as model 5 and model 4 get the best performance.

(4) For model 4 and model 5, the training term has better performance than test term. This may be due to the overlearning of SVM model.

(5) Although model 4 and model 5 have the best overall performance, they still show poor forecasting precision for high fire levels (they only precisely forecast $50 \%$ of fire level 4 for Hunan province, $12.5 \%$ of fire level 4 for Anhui province, and no fire level 3).

Thereafter, to estimate the effect of the human index on the performance of fire forecasting, the human index is added to all of the above 5 models. And these new 5 models are called model 1 with human index, model 2 with human index, model 3 with human index, model 4 with human index, and model 5 with human index, respectively. The results of these new 5 models are given in Tables 4 and 5. Similar to [19], Figure 5 shows the results of each trial related to models with the human index. From Tables 4 and 5, the following can be noted: 

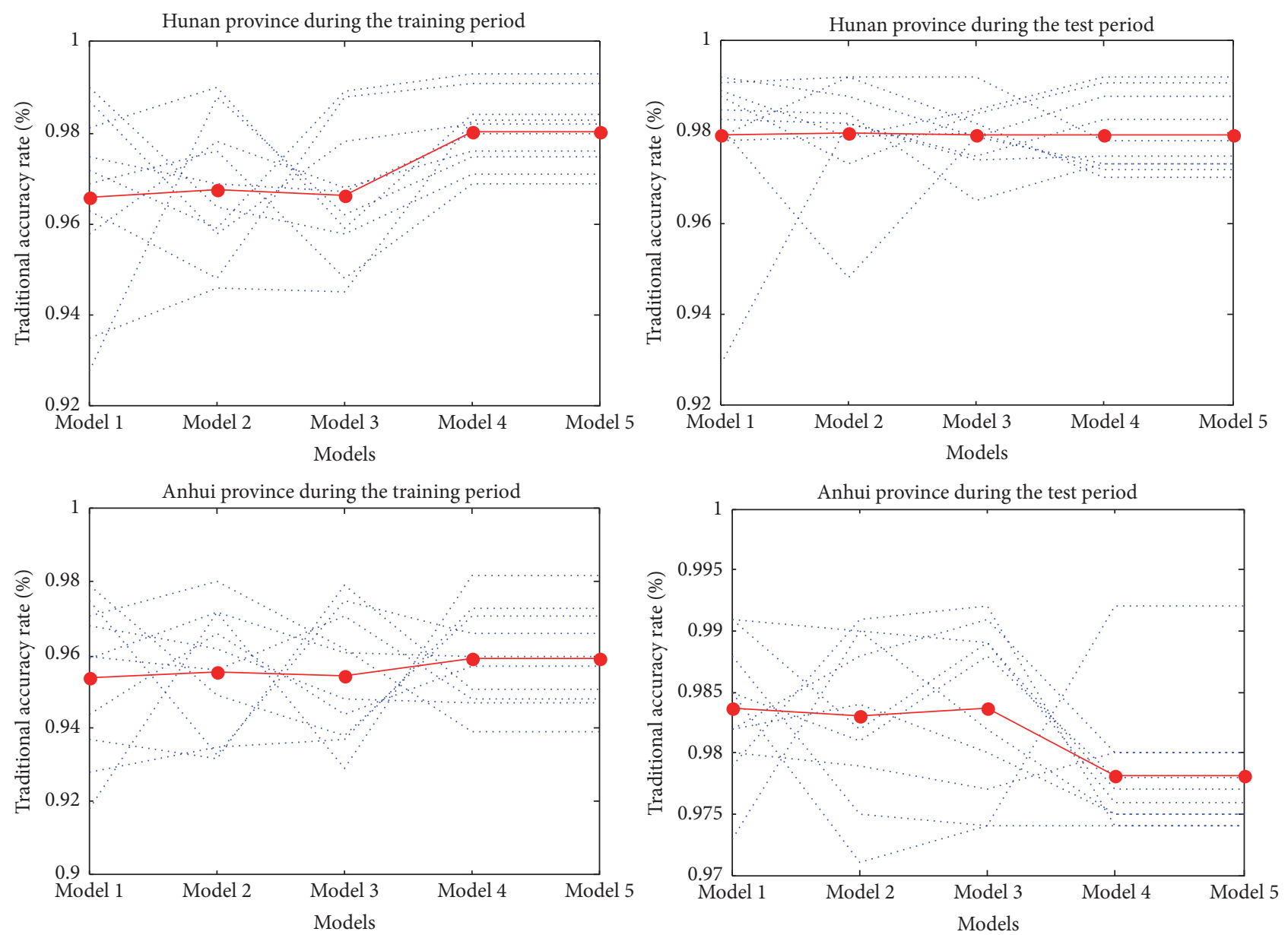

FIGURE 4: The results of each trial for models without the human index (the 10 trials are indicated by dashed lines, and the average performance is indicated by a solid line).

TABLE 4: The results of typical models with the human index in Hunan province.

\begin{tabular}{|c|c|c|c|c|c|c|}
\hline & Index & $\begin{array}{l}\text { Model } 1 \text { with } \\
\text { human index }\end{array}$ & $\begin{array}{l}\text { Model } 2 \text { with } \\
\text { human index }\end{array}$ & $\begin{array}{l}\text { Model } 3 \text { with } \\
\text { human index }\end{array}$ & $\begin{array}{l}\text { Model } 4 \text { with } \\
\text { human index }\end{array}$ & $\begin{array}{l}\text { Model } 5 \text { with } \\
\text { human index }\end{array}$ \\
\hline \multirow{6}{*}{ Training } & Traditional accuracy rate & 0.9795 & 0.9817 & 0.9822 & 0.9858 & 0.9849 \\
\hline & Weighted accuracy rate & 1.0406 & 1.0452 & 1.0488 & 1.0579 & 1.0561 \\
\hline & Traditional accuracy rate of fire level 1 & 1.0 & 1.0 & 1.0 & 1.0 & 1.0 \\
\hline & Traditional accuracy rate of fire level 2 & 0.1304 & 0.1956 & 0.1956 & 0.3261 & 0.2826 \\
\hline & Traditional accuracy rate of fire level 3 & 0.6667 & 0.8667 & 0.8667 & 1.0 & 1.0 \\
\hline & Traditional accuracy rate of fire level 4 & 1.0 & 0.9286 & 1.0 & 1.0 & 1.0 \\
\hline \multirow{6}{*}{ Test } & Traditional accuracy rate & 0.9836 & 0.9836 & 0.9845 & 0.9849 & 0.9836 \\
\hline & Weighted accuracy rate & 0.9986 & 0.9986 & 1.0014 & 1.0023 & 1.0005 \\
\hline & Traditional accuracy rate of fire level 1 & 1.0 & 1.0 & 1.0 & 1.0 & 0.9991 \\
\hline & Traditional accuracy rate of fire level 2 & 0.0303 & 0.0303 & 0.0606 & 0.0909 & 0.0606 \\
\hline & Traditional accuracy rate of fire level 3 & 0.6 & 0.6 & 0.7 & 0.7 & 0.7 \\
\hline & Traditional accuracy rate of fire level 4 & 1.0 & 1.0 & 1.0 & 1.0 & 1.0 \\
\hline
\end{tabular}



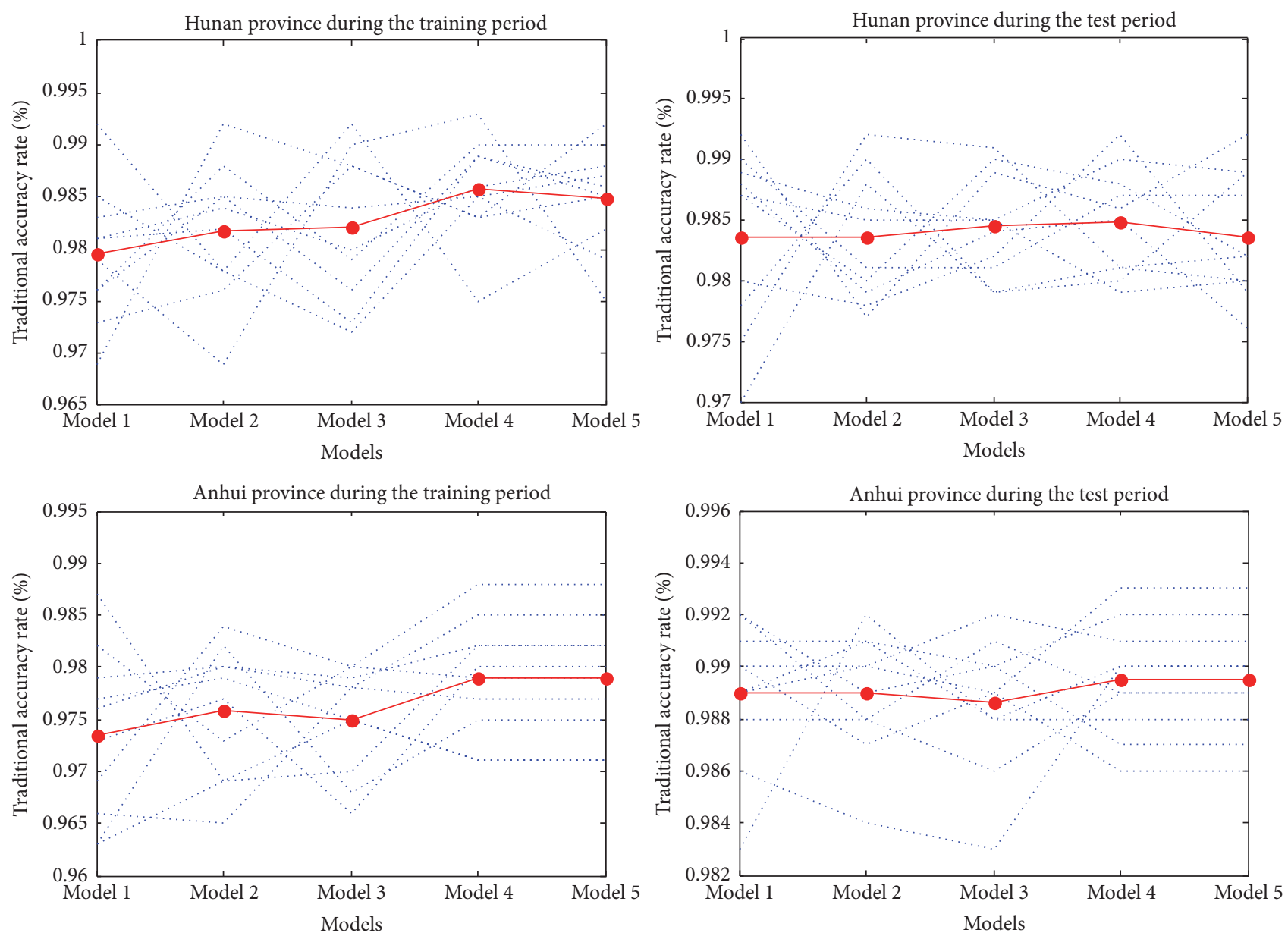

FIGURE 5: The results of each trial for models with the human index (the 10 trials are indicated by dashed lines, and the average performance is indicated by a solid line).

TABLE 5: The results of typical models with the human index in Anhui province.

\begin{tabular}{|c|c|c|c|c|c|c|}
\hline & Index & $\begin{array}{l}\text { Model } 1 \text { with } \\
\text { human index }\end{array}$ & $\begin{array}{l}\text { Model } 2 \text { with } \\
\text { human index }\end{array}$ & $\begin{array}{l}\text { Model } 3 \text { with } \\
\text { human index }\end{array}$ & $\begin{array}{l}\text { Model } 4 \text { with } \\
\text { human index }\end{array}$ & $\begin{array}{l}\text { Model } 5 \text { with } \\
\text { human index }\end{array}$ \\
\hline \multirow{6}{*}{ Training } & Traditional accuracy rate & 0.9735 & 0.9758 & 0.9749 & 0.9790 & 0.9790 \\
\hline & Weighted accuracy rate & 1.0625 & 1.0707 & 1.0652 & 1.0853 & 1.0853 \\
\hline & Traditional accuracy rate of fire level 1 & 1 & 1 & 1 & 1 & 1 \\
\hline & Traditional accuracy rate of fire level 2 & 0.2167 & 0.2667 & 0.2667 & 0.3 & 0.3 \\
\hline & Traditional accuracy rate of fire level 3 & 0.5 & 0.5714 & 0.5 & 0.7857 & 0.7857 \\
\hline & Traditional accuracy rate of fire level 4 & 0.8518 & 0.8889 & 0.8518 & 0.9630 & 0.9630 \\
\hline \multirow{6}{*}{ Test } & Traditional accuracy rate & 0.9890 & 0.9890 & 0.9886 & 0.9895 & 0.9895 \\
\hline & Weighted accuracy rate & 1.0146 & 1.0155 & 1.0146 & 1.0178 & 1.0178 \\
\hline & Traditional accuracy rate of fire level 1 & 1 & 1 & 1 & 0.9995 & 0.9995 \\
\hline & Traditional accuracy rate of fire level 2 & 0.1667 & 0.125 & 0.0833 & 0.1667 & 0.1667 \\
\hline & Traditional accuracy rate of fire level 3 & 0.25 & 0.5 & 0.5 & 0.75 & 0.75 \\
\hline & Traditional accuracy rate of fire level 4 & 0.875 & 0.875 & 0.875 & 0.875 & 0.875 \\
\hline
\end{tabular}


(1) The performance shown in Tables 4 and 5 is superior to that in Tables 2 and 3. With the inclusion of the human index, the fire forecasting precision has been significantly improved; the precision of high fire level especially (fire level 3 and fire level 4) is much enhanced.

(2) Similar to the results in Tables 2 and 3, model 2 with human index is superior to model 1 with human index. This is because the relative air humidity is necessary for fire forecasting.

(3) Different from the results in Table 2, model 3 with human index is slightly better than model 2 with human index for Hunan province. The reason may be that the fire forecasting model relies on more information about the daily mean meteorological condition with the inclusion of the human index. For Anhui province, model 2 with human index is better than model 3 with human index. This is consistent with Table 3. This may indicate that the potential predictors make different contribution for different provinces.

(4) For Hunan province, the calibration results of the predictor parameters of model 5 with human index show that the predictor "precipitation" should be excluded in the model inputs. For model 5 with human index, as the human index is formed by precipitation and human activities, thus the predictor "precipitation" is treated as to have duplicated information with the human index during the calibration of the predictor parameters.

(5) For Hunan province, the performance of model 5 with human index is slightly worse than of model 4 with human index. As the calibration results of model 4 can be treated as a special situation of model 5, this may indicate that the model calibration algorithm may be easily falling into local optimum. Developing a more efficient calibration algorithm is the next task we plan to do in the near future. This also demonstrates that all of the predictors, especially the human index, are necessary for fire forecasting. However, for Anhui province, model 5 has the same results as model 4 . This may be because the algorithm does not fall into local optimum for calibration of this model.

\section{Conclusions}

In our research, a fire prediction model is proposed incorporating meteorological data as well as human activities. And this model is applied in Hunan province and Anhui province, which seriously suffer from fire disasters. The results show that the model has good prediction precision and can be a powerful tool for practical application. However, the model calibration is also a very important part for fire forecasting. And developing an efficient and global optimization algorithm is the next work we plan to do in the near future.

\section{Competing Interests}

The authors declare no competing interests.

\section{Authors' Contributions}

Jiazheng Lu and Jun Guo conceived and designed the experiments; Li Yang performed the experiments; Tao Feng and Jie Zhang analyzed the data; Jun Guo wrote the paper.

\section{Acknowledgments}

The authors appreciate the support from the State Key Program of State Grid Corporation of China.

\section{References}

[1] R. E. Burgan, "Use of remotely sensed date for fire danger estimation," Remote Sensing of Environment, vol. 4, pp. 1-8, 1996.

[2] R. E. Burgan, R. W. Klaver, and J. M. Klarer, "Fuel models and fire potential from satellite and surface observations," International Journal of Wildland Fire, vol. 8, no. 3, pp. 159-170, 1998.

[3] C. Vega-Garcia, B. Lee, P. Woodard, and S. Titus, "Applying neural network technology to human-caused wildfire occurence prediction," AI Applications, vol. 10, pp. 9-18, 1996.

[4] G. Wen, X. Hou, and H. Chen, "The application of artifical nervous net to forecasting forest fire," Journal of Biomathematics, vol. 16, pp. 225-228, 2001.

[5] L. Hu, Z. Feng, and Y. Nie, "Forest fire prediction research based on VLBP neural network," Scientia Silvae Sinicae, vol. 42, pp. 155-158, 2006.

[6] Z. Fu, Q. Sun, Y. Cai, and E. Dai, "Research on forecasting model of forest fire based on Grey-system theory," Scientia Silvae Sinicae, vol. 38, pp. 95-100, 2002.

[7] D. Stojanova, P. Panov, A. Kobler, S. Dzeroski, and K. Taskova, "Learning to predict forest fires with different DataMining techniques," in Proceedings of the 9th International Multiconference Information Society, Ljubljana, Slovenia, 2006.

[8] P. Cortez and A. Morais, "A data mining approach to predict forest fires using meteorological data," in Proceedings of the 13th Portuguese Conference on Artificial Intelligence (EPIA '07), Guimarães, Portugal, 2007.

[9] R. Storn and K. Price, "Differential evolution-a simple and efficient adaptive scheme for global optimization over continuous spaces," Tech. Rep. TR-95-012, ICSI, Berkeley, Calif, USA, 1995.

[10] R. Storn and K. Price, "Differential evolution-a simple and efficient heuristic for global optimization over continuous spaces," Journal of Global Optimization, vol. 11, no. 4, pp. 341359, 1997.

[11] X. Yuan, Y. Zhang, L. Wang, and Y. Yuan, "An enhanced differential evolution algorithm for daily optimal hydro generation scheduling," Computers and Mathematics with Applications, vol. 55, no. 11, pp. 2458-2468, 2008.

[12] L. D. S. Coelho, J. G. Sauer, and M. Rudek, "Differential evolution optimization combined with chaotic sequences for image contrast enhancement," Chaos, Solitons \& Fractals, vol. 42, no. 1, pp. 522-529, 2009.

[13] H. I. Shaheen, G. I. Rashed, and S. J. Cheng, "Application of differential evolution algorithm for optimal location and 
parameters setting of UPFC considering power system security," European Transactions on Electrical Power, vol. 19, no. 7, pp. 911932, 2009.

[14] S. Das and S. Sil, "Kernel-induced fuzzy clustering of image pixels with an improved differential evolution algorithm," Information Sciences, vol. 180, no. 8, pp. 1237-1256, 2010.

[15] F. Neri and V. Tirronen, "Recent advances in differential evolution: a survey and experimental analysis," Artificial Intelligence Review, vol. 33, no. 1-2, pp. 61-106, 2010.

[16] Y. Lu, J. Zhou, Y. Li, and H. Qin, "Adaptive differential evolution algorithm combined with chaotic search," Computer Engineering and Applications Journal, vol. 44, pp. 31-33, 2008.

[17] L. Wu, Y. Wang, X. Yuan, and S. Zhou, "Differential evolution algorithm with adaptive second mutation," Control and Decision, vol. 21, pp. 117-120, 2006.

[18] J. Guo, J. Zhou, H. Wang, and Q. Zou, "Structure optimization and parameter calibration of empirical hydrological model under multi-objective framework," Journal of Hydroelectric Engineering, vol. 33, no. 2, pp. 1-7, 2014.

[19] S. Leva, A. Dolara, F. Grimaccia, M. Mussetta, and E. Ogliari, "Analysis and validation of 24 hours ahead neural network forecasting of photovoltaic output power," Mathematics and Computers in Simulation, vol. 131, pp. 88-100, 2017. 


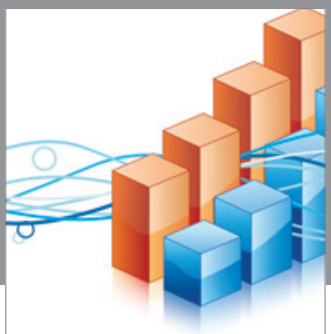

Advances in

Operations Research

vatem alat4

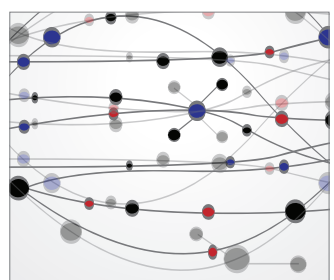

\section{The Scientific} World Journal
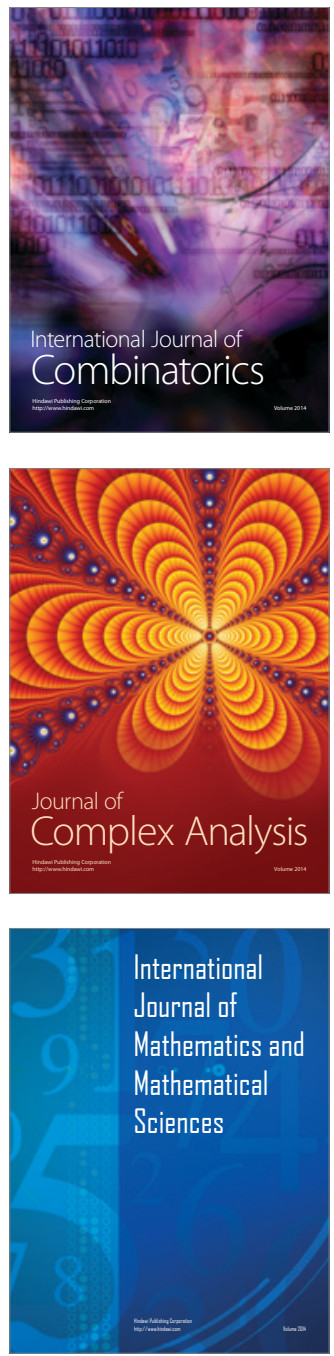
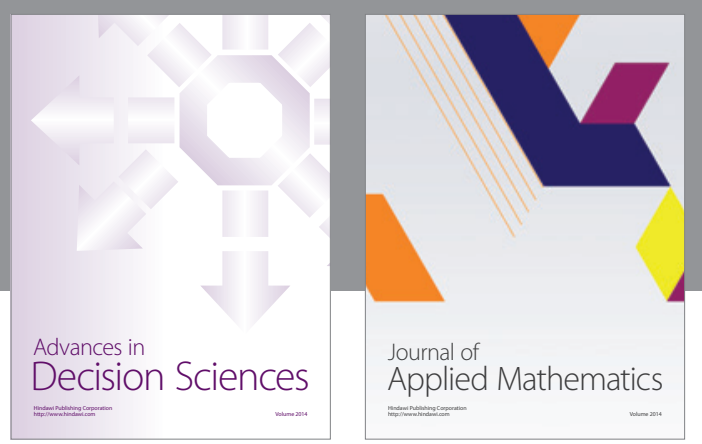

Algebra

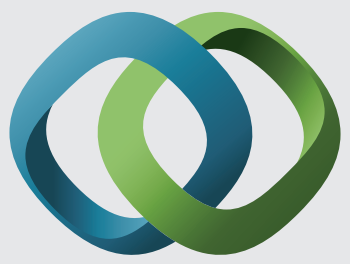

\section{Hindawi}

Submit your manuscripts at

http://www.hindawi.com
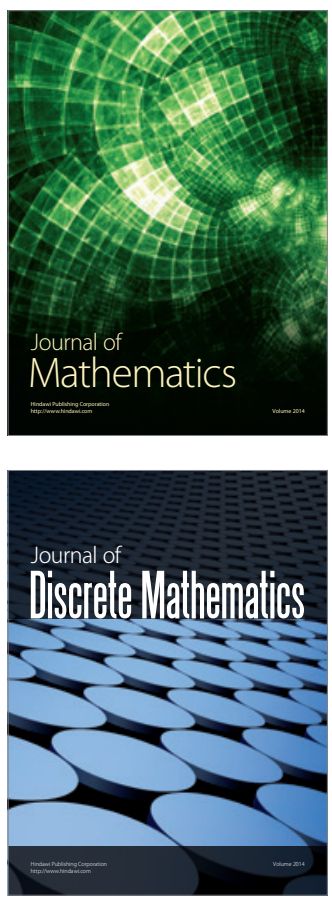

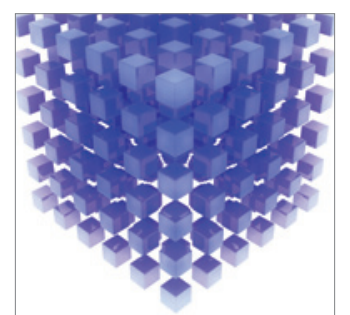

Mathematical Problems in Engineering
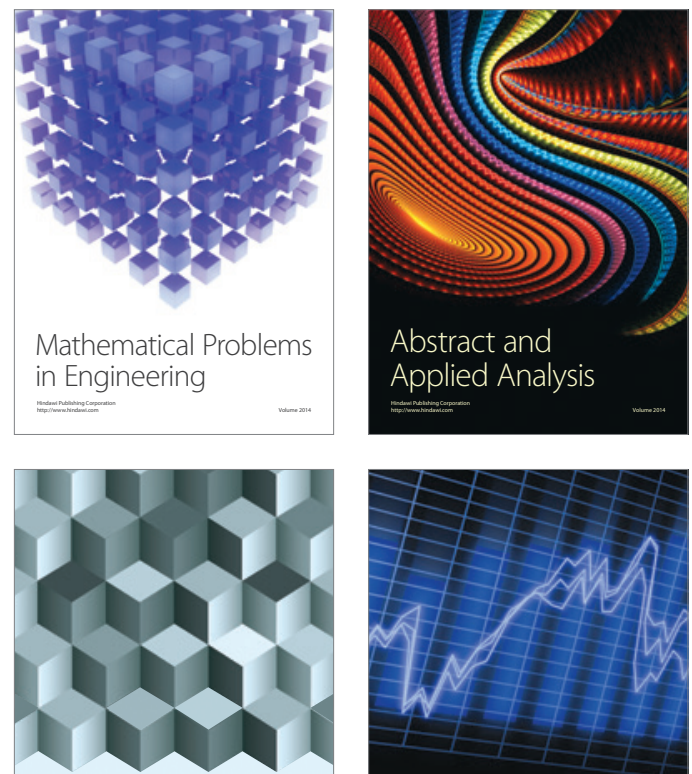

Journal of

Function Spaces

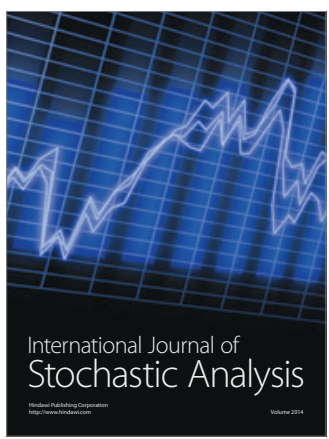

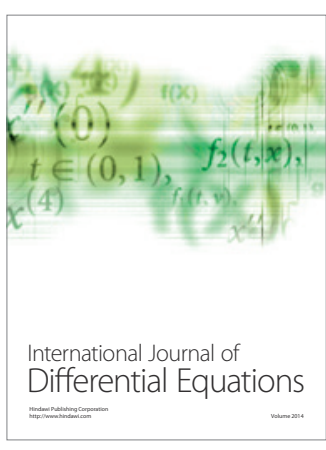
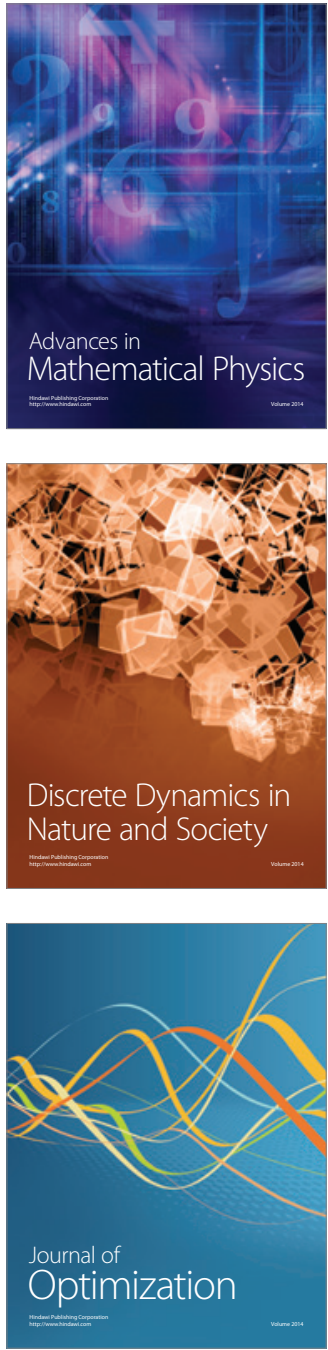